\section{Diuretic therapy}

\section{Mark Oyama}

Diuretic therapy is a mainstay of treatment of congestive heart failure in both the dog and the cat. Diuretics offer effective relief of congestion and clinical signs. Many clinicians have a great deal of experience and expertise in dosing commonly used agents such as frusemide. On the other hand, experience with newer or less common agents, such as torsemide, is now just emerging. The best use of diuretics balances relief of congestion with avoidance of complications such as electrolyte or renal enzyme imbalances.

In this regard, tailoring of diuretic therapy to the individual patient is crucial. As the course of clinical disease progresses and need for higher doses of diuretics increases, resistance to frusemide is becoming more and more recognized as an impediment to successful control of congestion. Often, dogs and cats receiving extremely high doses of frusemide continue to have clinical signs of congestion. In these instances, 'resetting' therapy through use of subcutaneous frusemide, alternate or adjunctive diuretic agents, or maximization of other non-diuretic medications can help restore diuresis and alleviate clinical signs.

\section{KEY LEARNING OBJECTIVES}

- Know the standard dosing regimens for treatment of 'typical' cases of acute and chronic heart failure in the dog and cat

- Know about the use of diuretics other than frusemide, such as the loop diuretic torsemide, the thiazide diuretic hydrochlorothiazide, and the aldosterone antagonist spironolactone

- Know how to modify drug and dosing in cases that have a poor response to conventional therapy

\section{MULTIPLE CHOICE QUESTIONS}

1. Which of the following strategies can help restore responsiveness of diuretics?

(A) Administering one of the daily frusemide doses as a subcutaneous injection

(B) Replacing frusemide with a different loop diuretic such as torsemide

(C) Adding a less potent but longer acting diuretic such as hydrochlorothiazide

(D) All of the above

2. Which of the following are potential reasons for 'diuretic resistance'?

(A) Poor oral bioavailability

(B) Underlying kidney disease

(C) Renal tubular hypertrophy

(D) All of the above

3. Which of the following statements regarding the loop diuretic torsemide is true?

(A) Torsemide is less potent than frusemide on a $\mathrm{mg}$ to $\mathrm{mg}$ basis

(B) Torsemide is free of any potential effects on renal enzymes or electrolytes

(C) Torsemide can be used to help restore diuresis in cases of refractory heart failure

(D) Torsemide has a shorter half-life than frusemide 